\title{
Mistura em Tanque de Boro e Herbicidas em Semeadura CONVENCIONAL DE GIRASSOL ${ }^{1}$
}

\author{
Tank-Mix of Boron and Herbicides on Conventional Sunflower Sowing
}

CASTRO, C. ${ }^{2}$, BRIGHENTI, A.M. ${ }^{2}$ e OLIVEIRA JÚNIOR, A. ${ }^{3}$

\begin{abstract}
RESUMO - Os objetivos deste experimento foi avaliar a resposta do girassol às aplicações de boro (B), isoladas ou em mistura com herbicidas, e o controle de plantas daninhas por meio de experimento conduzido na Embrapa Soja, Londrina-PR. Os tratamentos foram acetochlor (1,92 kg i.a. ha-1), oxyfluorfen $\left(0,36 \mathrm{~kg}\right.$ i.a. ha-1), sulfentrazone $\left(0,35 \mathrm{~kg}\right.$ i.a.ha $\left.{ }^{-1}\right)$, trifluralin (1,80 kg i.a. ha-1) e as testemunhas capinada e sem capina. Todos os tratamentos foram aplicados, isoladamente ou em mistura, com $2 \mathrm{~kg} \mathrm{ha}^{-1}$ de $\mathrm{B}\left(\mathrm{Na}_{2} \mathrm{~B}_{4} \mathrm{O}_{7} \cdot 10 \mathrm{H}_{2} \mathrm{O}\right.$ - bórax e $\mathrm{H}_{3} \mathrm{BO}_{3}$ - ácido bórico). O tratamento mais eficiente foi acetochlor mais ácido bórico; essa combinação resultou em solução mais homogênea da calda de pulverização, quando comparada com os herbicidas mais bórax. O herbicida acetochlor aplicado isoladamente ou em mistura com as duas fontes de B foi eficiente no controle da trapoeraba (Commelina benghalensis), do picãopreto (Bidens pilosa) e da corda-de-viola (Ipomoea grandifolia). Os herbicidas oxyfluorfen e sulfentrazone, aplicados isoladamente ou em misturas com as duas fontes de B, foram eficientes no controle do amendoim-bravo (Euphorbia heterophylla) e da corda-de-viola, respectivamente. É viável a aplicação de boro juntamente com os herbicidas testados nesta pesquisa em mistura em tanque, evitando a deficiência desse micronutriente e controlando as plantas daninhas na cultura do girassol.
\end{abstract}

Palavras-chave: Helianthus annuus, controle químico, plantas daninhas, micronutriente.

\begin{abstract}
The objectives of this study were to determine the response of sunflower to application of boron (B) fertilizer and herbicides, alone or in combination, and to determine its weed control. A field experiment was carried out at Embrapa Soja, Londrina, Parana, Brazil. The treatments were acetochlor (1.92 $\mathrm{kg}$ a.i. $\left.\mathrm{ha}^{-1}\right)$, oxyfluorfen $\left(0.36 \mathrm{~kg}\right.$ a.i. ha $\left.\mathrm{a}^{-1}\right)$, sulfentrazone $\left(0.35 \mathrm{~kg}\right.$ a.i. $\left.\mathrm{ha}^{-1}\right)$, trifluralin (1.80 kg a.i. ha $)$ and two controls (with and without hoeing). All treatments were applied alone or in combination with $2 \mathrm{~kg} \mathrm{ha}^{-1}$ of $\mathrm{B}\left(\mathrm{Na}_{2} \mathrm{~B}_{4} \mathrm{O}_{7} \cdot 1 \mathrm{OH}_{2} \mathrm{O}-\right.$ borax and $\mathrm{H}_{3} \mathrm{BO}_{3}$ - boric acid). Acetochlor plus boric acid was the most efficient treatment. Herbicides mixed with boric acid provided a more homogeneous aqueous suspension when compared with herbicides plus borax. Acetochlor applied alone or in combination with two B sources was efficient in controlling wandering jew (Commelina benghalensis), hairy beggarticks (Bidens pilosa) and morning glory (Ipomoea grandifolia). Oxyfluorfen and sulfentrazone applied alone or in combination with the $B$ sources were efficient in controlling wild poinsettia (Euphorbia heterophylla) and morning glory (Ipomoea grandifolia), respectively. The application of $B$ with herbicides in tank-mixes is a good strategy for avoiding B deficiency and controlling weeds in sunflower crop.
\end{abstract}

Key words: Helianthus annuus, chemical control, weeds, micronutrient.

Recebido para publicação em 6/8/2001 e na forma revisada em 15/2/2002.

Embrapa Soja, Caixa postal 231, 86970-001 Londrina-PR; ${ }^{3}$ Embrapa Soja, Bolsista CNPq.

Planta Daninha, Viçosa-MG, v.20, p.83-91, 2002 


\section{INTRODUÇÃO}

O girassol (Helianthus annuus L.) é uma dicotiledônea anual, pertencente à família Asteraceae. É originário da América do Norte e cultivado nos cinco continentes, totalizando mais de 22 milhões de hectares. No Brasil, seu cultivo ocupa uma área em torno de 90.000 ha, principalmente na região dos Cerrados (Brighenti, 2000).

Para obter alta produtividade de girassol, é necessário boa fertilidade do solo, principalmente de boro (B). Níveis baixos desse micronutriente levam ao aparecimento de sintomas de deficiência nas plantas, notadamente nas fases de florescimento e maturação (Castro et al., 1997). Os sintomas de deficiência de B também estão associados aos períodos de estresse hídrico, comuns às regiões produtoras de girassol na época de "safrinha", na região dos Cerrados.

O boro é um elemento de pouca mobilidade na planta e, conseqüentemente, os sintomas de deficiência se manifestam, primeiramente, nos tecidos jovens (Calle-Manzano, 1985), podendo ocorrer a morte do ápice dos brotos e das raízes, levando ao aparecimento de brotações laterais (Gil Martinez, 1995). As plantas afetadas apresentam formação típica de roseta, com as folhas enrugadas, os pecíolos e os talos rompidos, e as flores, geralmente, não se formam.

A aplicação de B via solo, através de adubos granulados contendo o micronutriente, apresenta a desvantagem de ocorrer segregação entre a fonte de $\mathrm{B}$ e os componentes do fertilizante durante a mistura e o manuseio. A segregação interfere na uniformidade de aplicação de boro no solo, devido, principalmente, às baixas quantidades desse micronutriente a serem aplicadas (Mortvedt \& Woodruff, 1993).

A aplicação foliar do boro é viável, porém é necessário repetir as pulverizações, em razão da baixa mobilidade desse elemento dos tecidos mais velhos para os novos (Anderson \& Ohki, 1972). Além disso, essa operação aumenta os custos de produção e compacta o solo, com possibilidade de quebra das plantas.

Outro fator que interfere na produção do girassol é a ocorrência de plantas daninhas.
Muitos autores têm medido a magnitude desses danos, chegando a valores entre 20 e $50 \%$ de perda de rendimento de grãos (Blamey et al., 1997). A presença de invasoras durante as primeiras etapas de cultivo do girassol resulta em plantas cloróticas, de menor porte, com severa diminuição da área foliar, do diâmetro do caule e do capítulo (Blamey et al., 1997).

O objetivo deste experimento foi avaliar a resposta do girassol às aplicações de boro, isoladas ou em mistura com herbicidas, bem como o controle de plantas daninhas.

\section{MATERIAL E MÉTODOS}

O experimento foi conduzido na Embrapa Soja, Londrina-PR. O solo da área é classificado como Latossolo Roxo eutrófico, com $775 \mathrm{~g} \mathrm{~kg}^{-1}$ de argila, $181 \mathrm{~g} \mathrm{~kg}^{-1}$ de silte, $44 \mathrm{~g} \mathrm{~kg}^{-1}$ de areia, $19,8 \mathrm{~g} \mathrm{dm}^{-3}$ de C e 0,27 $\mathrm{mg} \mathrm{dm}^{-3}$ de B.

O delineamento experimental utilizado foi o de blocos casualizados, com cinco repetições. Os tratamentos foram constituídos dos herbicidas acetochlor $(1,92 \mathrm{~kg}$ i.a. ha-1), oxyfluorfen $(0,36 \mathrm{~kg}$ i.a.ha-1), sulfentrazone $(0,35 \mathrm{~kg}$ i.a. ha-1), trifluralin $(1,80 \mathrm{~kg}$ i.a. ha-1) e as testemunhas capinada e sem capina. Todos os tratamentos foram aplicados em préemergência do girassol, isolados ou em mistura, com $2 \mathrm{~kg} \mathrm{ha}^{-1}$ de boro, utilizando duas fontes de $\mathrm{B}$ (bórax - $\mathrm{Na}_{2} \mathrm{~B}_{4} \mathrm{O}_{7} \cdot 1 \mathrm{H}_{2} \mathrm{O}$ com $11,5 \%$ de $\mathrm{B}$ e ácido bórico - $\mathrm{H}_{3} \mathrm{BO}_{3}$ com $17,5 \%$ de B) (Tabela 1).

O híbrido de girassol Cargill 11 foi semeado no espaçamento de $0,70 \mathrm{~m}$, com três plantas por metro linear. As parcelas foram constituídas por quatro linhas de girassol com sete metros de comprimento, com área total de 19,6 $\mathrm{m}^{2}$ e área útil de $8,4 \mathrm{~m}^{2}$.

Na aplicação dos tratamentos foi utilizado pulverizador costal, a pressão constante, mantida por $\mathrm{CO}_{2}$ comprimido de $276 \mathrm{kPa}$, equipado com barra de $2,5 \mathrm{~m}$ de largura e seis bicos de jato plano 11002 XP2, com volume de pulverização equivalente a $250 \mathrm{~L} \mathrm{ha}^{-1}$. Por ocasião da aplicação dos produtos, a temperatura do ambiente era de $28{ }^{\circ} \mathrm{C}$ e a umidade relativa do ar, de $86 \%$, com solo úmido na superfície e velocidade do vento de $4 \mathrm{~km} \mathrm{~h}^{-1}$.

Foram avaliados o controle por espécie de plantas daninhas e o controle geral aos 25 e 
35 dias após a aplicação (DAA), utilizando a escala visual de $0 \%$ - 100\%, em que 0\% representou nenhum controle e $100 \%$, o controle total. Para análise de tecido, foi coletada a folha mais jovem e completamente expandida das plantas da área útil de cada parcela (estádio R4/R5). Foram obtidos os valores médios da altura das plantas de girassol, do diâmetro do capítulo, do número de aquênios por capítulo e do peso de mil aquênios. A produtividade foi obtida das plantas da área útil de cada parcela.

Os resultados obtidos foram submetidos à análise de variância e as médias comparadas pelo teste de Duncan a $5 \%$ de probabilidade.

\section{RESULTADOS E DISCUSSÃO}

O ácido bórico dissolvido em água, à temperatura ambiente, proporcionou mistura homogênea com os herbicidas. O bórax apresentou maior dificuldade de dissolução, em razão da sua menor solubilidade (Weast \& Astle 1982). Dessa forma, foi necessário o aquecimento prévio da água e agitação, para evitar o surgimento de precipitado e a possibilidade de entupimento dos bicos do pulverizador.
Nas misturas de herbicidas mais bórax, foi observada pequena formação de solução mais densa, à semelhança de "nata", que se depositou na parte inferior do reservatório do pulverizador. Entretanto, essa formação não afetou a passagem da calda pelos bicos de pulverização nem o controle das plantas daninhas. Isso pode ter ocorrido em função da diferença entre o pH do bórax, que é alcalino, e as caldas de pulverização dos herbicidas, que são ácidas (Tabela 1).

O tratamento com o herbicida acetochlor (1,92 kg i.a. ha-1) mais ácido bórico (ACE+AB) proporcionou a maior produtividade (1.979 $\mathrm{kg} \mathrm{ha}^{-1}$ ), estatisticamente superior aos tratamentos com o herbicida trifluralin e aos tratamentos SUL + $\mathrm{BO}, \mathrm{SUL}+\mathrm{AB}, \mathrm{OXY}, \mathrm{OXY}+\mathrm{AB}, \mathrm{TC}+\mathrm{AB}$, TSC e TSC + BO (Tabela 2). Esse resultado demonstra a possibilidade da mistura de ácido bórico em tanque e a adequação da dose do acetochlor para o girassol. Brighenti et al. (2000b), trabalhando com quatro doses de acetochlor em girassol, que variaram de 1,15 a 4,6 $\mathrm{kg} \mathrm{ha}^{-1}$, verificaram que doses equivalentes ou superiores a $2,3 \mathrm{~kg}^{\text {ha-1 }}$ causaram reduções na produtividade e no teor de óleo da cultura.

Tabela 1 - Nomes técnicos dos herbicidas mais fontes de boro, doses aplicadas, pH da calda de pulverização e sigla utilizada para designar os tratamentos. Embrapa Soja, Londrina-PR, 2000

\begin{tabular}{|c|c|c|c|c|}
\hline Tratamentos & Nomes técnicos + fontes de boro & $\begin{array}{c}\text { Doses } \\
\left(\mathrm{kg} \mathrm{i.a.} \mathrm{ha}^{-1}\right)\end{array}$ & $\begin{array}{c}\mathrm{pH} \\
\text { calda }\end{array}$ & Sigla \\
\hline 01 & Acetochlor $^{1 /}$ & 1,92 & 4,87 & $\overline{\mathrm{ACE}}$ \\
\hline 02 & Acetochlor $+\mathrm{Na}_{2} \mathrm{~B}_{4} \mathrm{O}_{7} \cdot 10 \mathrm{H}_{2} 0^{\frac{5}{\prime}}$ & $1,92+2$ & 8,38 & $\mathrm{ACE}+\mathrm{BO}$ \\
\hline 03 & Acetochlor $+\mathrm{H}_{3} \mathrm{BO}_{3}{ }^{6}$ & $1,92+2$ & 4,45 & $\mathrm{ACE}+\mathrm{AB}$ \\
\hline 04 & Oxyfluorfen ${ }^{2 /}$ & 0,36 & 4,63 & OXY \\
\hline 05 & Oxyfluorfen $+\mathrm{Na}_{2} \mathrm{~B}_{4} \mathrm{O}_{7} \cdot 10 \mathrm{H}_{2} \mathrm{O}$ & $0,36+2$ & 8,85 & $\mathrm{OXY}+\mathrm{BO}$ \\
\hline 06 & Oxyfluorfen $+\mathrm{H}_{3} \mathrm{BO}_{3}$ & $0,36+2$ & 4,30 & $\mathrm{OXY}+\mathrm{AB}$ \\
\hline 07 & Sulfentrazone $\mathrm{e}^{3 /}$ & 0,35 & 4,57 & SUL \\
\hline 08 & Sulfentrazone $+\mathrm{Na}_{2} \mathrm{~B}_{4} \mathrm{O}_{7} .10 \mathrm{H}_{2} \mathrm{O}$ & $0,35+2$ & 8,86 & $\mathrm{SUL}+\mathrm{BO}$ \\
\hline 09 & Sulfentrazone $+\mathrm{H}_{3} \mathrm{BO}_{3}$ & $0,35+2$ & 4,32 & $\mathrm{SUL}+\mathrm{AB}$ \\
\hline 10 & Trifluralin $^{4 /}$ & 1,8 & 4,70 & TRI \\
\hline 11 & Trifluralin $+\mathrm{Na}_{2} \mathrm{~B}_{4} \mathrm{O}_{7} \cdot 10 \mathrm{H}_{2} \mathrm{O}$ & $1,8+2$ & 8,90 & TRI+BO \\
\hline 12 & Trifluralin $+\mathrm{H}_{3} \mathrm{BO}_{3}$ & $1,8+2$ & 4,35 & $\mathrm{TRI}+\mathrm{AB}$ \\
\hline 13 & Testemunha sem capina & - & - & TSC \\
\hline 14 & Testemunha sem capina $+\mathrm{Na}_{2} \mathrm{~B}_{4} \mathrm{O}_{7} \cdot 10 \mathrm{H}_{2} \mathrm{O}$ & 2 & 9,20 & $\mathrm{TSC}+\mathrm{BO}$ \\
\hline 15 & Testemunha sem capina $+\mathrm{H}_{3} \mathrm{BO}_{3}$ & 2 & 4,10 & $\mathrm{TSC}+\mathrm{AB}$ \\
\hline 16 & Testemunha capinada & - & - & $\mathrm{TC}$ \\
\hline 17 & Testemunha capinada $+\mathrm{Na}_{2} \mathrm{~B}_{4} \mathrm{O}_{7} \cdot 10 \mathrm{H}_{2} 0$ & 2 & 9,20 & $\mathrm{TC}+\mathrm{BO}$ \\
\hline 18 & Testemunha capinada $+\mathrm{H}_{3} \mathrm{BO}_{3}$ & 2 & 4,10 & $\mathrm{TC}+\mathrm{AB}$ \\
\hline
\end{tabular}

${ }^{1 /}$ Acetochlor (Surpass - $768 \mathrm{~g} \mathrm{~L}^{-1}$ ); ${ }^{2}$ oxyfluorfen (Galigan - $240 \mathrm{~g} \mathrm{~L}^{-1}$ ); ${ }^{3 /}$ sulfentrazone (Boral - $500 \mathrm{~g} \mathrm{~L}^{-1}$ ); ${ }^{4 /}$ trifluralin $($ Premerlin $600 \mathrm{~g} \mathrm{~L}^{-1}$ ); ${ }^{5 /} \mathrm{Na}_{2} \mathrm{~B}_{4} \mathrm{O}_{7} \cdot 10 \mathrm{H}_{2} \mathrm{O}$ (bórax-11,5\% de boro); ${ }^{6 /} \mathrm{H}_{3} \mathrm{BO}_{3}$ (ácido bórico - 17,5\% de boro). 
Apesar de não terem ocorrido diferenças significativas entre os tratamentos, os teores médios de $B$ nas folhas variaram de 48,2 $\mathrm{mg} \mathrm{dm}^{-3}$, no tratamento $\mathrm{OXY}+\mathrm{BO}$, a $31,1 \mathrm{mg} \mathrm{dm}^{-3}$, no tratamento $\mathrm{SUL}+\mathrm{AB}$ (Tabela 2). Reuter et al. (1997) estabeleceram como adequados os teores de boro de 40 a 250 $\mathrm{mg} \mathrm{dm}{ }^{-3} \mathrm{em}$ folhas de girassol colhidas no estádio $\mathrm{R}_{4} / \mathrm{R}_{5}$. Sfredo \& Sarruge (1990) determinaram o intervalo de 88 a $102 \mathrm{mg} \mathrm{dm}^{-3} \mathrm{de} \mathrm{B}$, no início do florescimento, como adequado para o girassol. Por outro lado, Blamey et al. (1979) estabeleceram o nível crítico de $34 \mathrm{mg} \mathrm{dm}^{-3}$, em folhas de girassol colhidas no início do florescimento de dois híbridos cultivados no campo. Apesar de os teores de alguns tratamentos serem enquadrados como adequados, foram observados sintomas de deficiência do nutriente em todos os tratamentos. Os principais sintomas da deficiência e de mais fácil percepção foram o aparecimento de manchas marrons no caule, logo abaixo do pecíolo das folhas próximas ao capítulo, seguidas de deformação, bronzeamento e espessamento do limbo foliar.

A baixa resposta nos teores de B nas folhas, em função da aplicação do nutriente, pode ser entendida pela pluviosidade ocorrida durante a condução do experimento (Figura 1). Do início do florescimento ao final do enchimento de aquênios, a pluviosidade foi de $44 \mathrm{~mm}$. Nesse período, as plantas receberam, aproximadamente, $20 \mathrm{~mm}$ de água através de irrigação por aspersão. Assim, apesar da adição de boro no solo, a falta de umidade afetou, seriamente, a sua disponibilidade e absorção pelas plantas. Para Gupta (1993), a umidade do solo parece afetar a disponibilidade de B mais do que qualquer outro nutriente. A quantidade reduzida de solução do solo e a conseqüente redução do fluxo de massa, assim como o limitado fluxo transpiratório durante o período seco, podem ter sido os fatores determinantes da deficiência de boro, apesar do fornecimento desse nutriente no solo.

Tabela 2 - Valores médios de altura de plantas $(\mathrm{cm})$, diâmetros de capítulo $(\mathrm{cm})$, número de aquênios por capítulo, peso de mil aquênios $(\mathrm{g})$, produtividade da cultura $\left(\mathrm{kg} \mathrm{ha}^{-1}\right)$ e teor de boro nas folhas de girassol $\left(\mathrm{mg} \mathrm{dm}{ }^{-3}\right)$, em função dos tratamentos. Embrapa Soja, Londrina-PR, 2000

\begin{tabular}{|c|c|c|c|c|c|c|}
\hline Tratamentos & $\begin{array}{c}\text { Altura de } \\
\text { plantas }\end{array}$ & $\begin{array}{c}\text { Diâmetro do } \\
\text { capítulo }\end{array}$ & $\begin{array}{c}\text { Número de } \\
\text { aquênios }\end{array}$ & $\begin{array}{c}\text { Peso de mil } \\
\text { aquênios }\end{array}$ & Produtividade & Teor de boro \\
\hline ACE & $143,94 \mathrm{ab}$ & $16,20 \mathrm{abcd}$ & $1.010 \mathrm{abc}$ & $39,76 \mathrm{ab}$ & $1.724,2 \mathrm{abc}$ & $46,4 \mathrm{a}$ \\
ACE + BO & $154,28 \mathrm{a}$ & $17,20 \mathrm{ab}$ & $1.113 \mathrm{ab}$ & $40,16 \mathrm{ab}$ & $1.926,8 \mathrm{ab}$ & $45,5 \mathrm{a}$ \\
ACE + AB & $148,50 \mathrm{ab}$ & $17,38 \mathrm{a}$ & $1.119 \mathrm{a}$ & $40,30 \mathrm{ab}$ & $1.979,2 \mathrm{a}$ & $46,5 \mathrm{a}$ \\
OXY & $149,24 \mathrm{ab}$ & $16,38 \mathrm{abc}$ & $1.012 \mathrm{abc}$ & $36,54 \mathrm{ab}$ & $1.589,2 \mathrm{bc}$ & $41,6 \mathrm{a}$ \\
OXY + BO & $149,18 \mathrm{ab}$ & $15,16 \mathrm{~cd}$ & $1.056 \mathrm{abc}$ & $36,80 \mathrm{ab}$ & $1.660,2 \mathrm{abc}$ & $48,2 \mathrm{a}$ \\
OXY + AB & $150,20 \mathrm{ab}$ & $15,68 \mathrm{abcd}$ & $916 \mathrm{abc}$ & $41,10 \mathrm{a}$ & $1.595,2 \mathrm{bc}$ & $45,9 \mathrm{a}$ \\
SUL & $142,14 \mathrm{ab}$ & $16,04 \mathrm{abcd}$ & $997 \mathrm{abc}$ & $39,26 \mathrm{ab}$ & $1.669,8 \mathrm{abc}$ & $37,8 \mathrm{a}$ \\
SUL + BO & $145,80 \mathrm{ab}$ & $15,66 \mathrm{abcd}$ & $971 \mathrm{abc}$ & $38,14 \mathrm{ab}$ & $1.584,8 \mathrm{bc}$ & $35,0 \mathrm{a}$ \\
SUL + AB & $138,62 \mathrm{~b}$ & $15,38 \mathrm{bcd}$ & $964 \mathrm{a}$ & $38,40 \mathrm{ab}$ & $1.589,2 \mathrm{bc}$ & $31,1 \mathrm{a}$ \\
TRI & $146,48 \mathrm{ab}$ & $14,36 \mathrm{~d}$ & $909 \mathrm{bc}$ & $37,88 \mathrm{ab}$ & $1.478,6 \mathrm{c}$ & $39,7 \mathrm{a}$ \\
TRI + BO & $146,76 \mathrm{ab}$ & $16,16 \mathrm{abcd}$ & $879 \mathrm{c}$ & $40,02 \mathrm{ab}$ & $1.502,0 \mathrm{c}$ & $34,5 \mathrm{a}$ \\
TRI + AB & $142,56 \mathrm{ab}$ & $14,90 \mathrm{~cd}$ & $902 \mathrm{c}$ & $37,14 \mathrm{ab}$ & $1.443,0 \mathrm{c}$ & $41,3 \mathrm{a}$ \\
TSC & $140,46 \mathrm{ab}$ & $15,80 \mathrm{abcd}$ & $953 \mathrm{abc}$ & $39,36 \mathrm{ab}$ & $1.614,6 \mathrm{bc}$ & $34,4 \mathrm{a}$ \\
TSC + BO & $146,88 \mathrm{ab}$ & $15,44 \mathrm{bcd}$ & $986 \mathrm{abc}$ & $36,42 \mathrm{~b}$ & $1.537,0 \mathrm{c}$ & $39,3 \mathrm{a}$ \\
TSC + AB & $146,62 \mathrm{ab}$ & $15,32 \mathrm{bcd}$ & $997 \mathrm{abc}$ & $38,12 \mathrm{ab}$ & $1.638,2 \mathrm{abc}$ & $33,5 \mathrm{a}$ \\
TC & $154,36 \mathrm{a}$ & $16,34 \mathrm{abc}$ & $1.050 \mathrm{abc}$ & $37,08 \mathrm{ab}$ & $1.662,6 \mathrm{abc}$ & $40,5 \mathrm{a}$ \\
TC + BO & $153,36 \mathrm{a}$ & $16,18 \mathrm{abcd}$ & $1.013 \mathrm{abc}$ & $38,36 \mathrm{ab}$ & $1.669,4 \mathrm{abc}$ & $39,1 \mathrm{a}$ \\
TC + AB & $145,82 \mathrm{ab}$ & $15,58 \mathrm{abcd}$ & $978 \mathrm{abc}$ & $37,38 \mathrm{ab}$ & $1.573,0 \mathrm{bc}$ & $38,6 \mathrm{a}$ \\
\hline CV $\%)$ & 6,37 & 7,89 & 13,79 & 7,86 & 14,74 & 32,29 \\
\hline
\end{tabular}

Médias seguidas pelas mesmas letras, nas colunas, não diferem estatisticamente entre si a 5\% de probabilidade pelo teste de Duncan. 


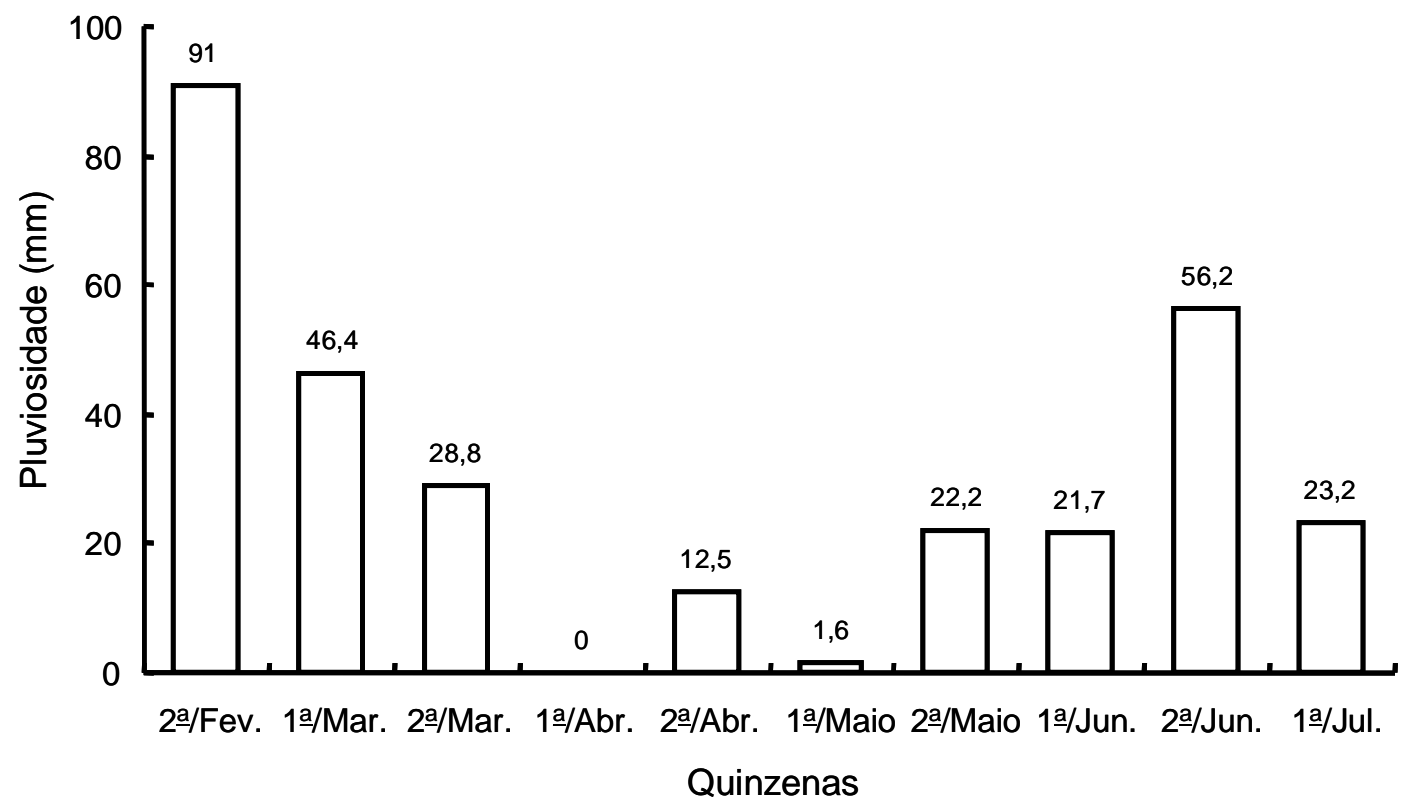

Figura 1 - Pluviosidade quinzenal ocorrida no período de fevereiro a julho de 2000, em Londrina-PR. Embrapa Soja, Londrina-PR, 2000.

A combinação do número de aquênios por capítulo e do peso de mil aquênios é importante para a composição final do rendimento da lavoura de girassol. Assim, observa-se, na Tabela 2, que os tratamentos $\mathrm{ACE}+\mathrm{BO}$ e $\mathrm{ACE}$ $+\mathrm{AB}$, com maior produtividade, foram aqueles com os maiores valores para esses dois parâmetros.

A redução do número de aquênios por capítulo pode estar associada ao estresse hídrico ocorrido durante o florescimento (Figura 1). Para Connor \& Hall (1997), o estresse hídrico reduz a expansão celular, a extrusão das anteras, a deiscência do pólen, a extensão do estigma e a sua receptividade, contribuindo para a infertilidade. Além da redução do número de aquênios, a continuidade do estresse hídrico durante o enchimento de aquênios reduziu, também, o peso de mil aquênios e, conseqüentemente, a produtividade.

Na Figura 2, observa-se o elevado coeficiente de correlação entre o número de aquênios e a produtividade $\left(\mathrm{r}=0,90^{* *}\right)$, evidenciando que esse parâmetro é adequado para avaliar a produtividade do girassol.

Os tratamentos $\mathrm{ACE}+\mathrm{BO}, \mathrm{TC}$ e $\mathrm{TC}+\mathrm{BO}$ proporcionaram as maiores alturas de plantas (Tabela 2). A média geral desse parâmetro foi de $147 \mathrm{~cm}$, valor abaixo da média de $196 \mathrm{~cm}$, obtido para o híbrido Cargill 11 (Embrapa Soja, 1999). A redução da altura das plantas, provavelmente, foi conseqüência do estresse hídrico durante o período experimental.

Para o diâmetro de capítulo, o tratamento $\mathrm{ACE}+\mathrm{AB}$ foi superior aos tratamentos TRI, $\mathrm{TRI}+\mathrm{AB}, \mathrm{OXY}+\mathrm{BO}, \mathrm{SUL}+\mathrm{AB}, \mathrm{TSC}+\mathrm{BO} \mathrm{e}$ TSC + AB (Tabela 2).

Nas avaliações da produtividade e do número de aquênios por capítulo, observou-se correlação alta e positiva entre a produtividade e o diâmetro do capítulo $(\mathrm{r}=0,81)$ e entre o diâmetro do capítulo e o número de aquênios por capítulo $(r=0,68)$ (Tabela 3). Essas correlações demonstram a importância do peso de mil aquênios, do número de aquênios por capítulo e do diâmetro do capítulo na produtividade dessa cultura.

Com relação ao controle de plantas daninhas, os tratamentos que receberam o herbicida acetochlor $(\mathrm{ACE}, \mathrm{ACE}+\mathrm{BO}$ e $\mathrm{ACE}+\mathrm{AB})$ foram eficientes no controle da trapoeraba (Commelina benghalensis), atingindo valores próximos a $100 \%$ aos 25 e 35 DAA (Tabela 4), não diferindo da testemunha capinada (TC). O nível de controle dos tratamentos evidencia não só a eficiência do acetochlor no controle dessa 
invasora, como também a possibilidade de combinação desse herbicida com bórax ou ácido bórico.

Os tratamentos com os herbicidas oxyfluorfen e sulfentrazone apresentaram controle mediano da trapoeraba aos 25 e 35 DAA.

Os tratamentos contendo o trifluralin não foram eficientes no controle dessa espécie, assemelhando-se à testemunha sem capina (TSC). Neste experimento, as espécies daninhas de "folhas largas" predominaram, havendo baixa incidência de invasoras de "folhas estreitas". Dessa maneira, todas as parcelas que receberam esse herbicida, isoladamente ou em mistura com as duas fontes de B, obtiveram valores baixos de percentagem de controle.
Os tratamentos $\mathrm{ACE}, \mathrm{ACE}+\mathrm{BO}$ e $\mathrm{ACE}+$ $\mathrm{AB}$ proporcionaram niveis de controle do picãopreto (Bidens pilosa) próximos a $100 \%$, nas duas avaliações. Em experimento conduzido por Brighenti et al. (2000a), foi obtido o controle de $87 \%$ do picão-preto, com a dose de $2,1 \mathrm{~kg} \mathrm{ha}^{-1}$ do acetochlor.

Os tratamentos com os herbicidas oxyfluorfen e sulfentrazone apresentaram eficiência intermediária, controlando cerca de 50 e $60 \%$ da infestação aos 25 e 35 DAA, respectivamente.

Assim como no controle da trapoeraba, os resultados demonstram não só a eficiência do herbicida acetochlor no controle do picão-preto, como também a possibilidade de combinação desse herbicida com as duas fontes de B.

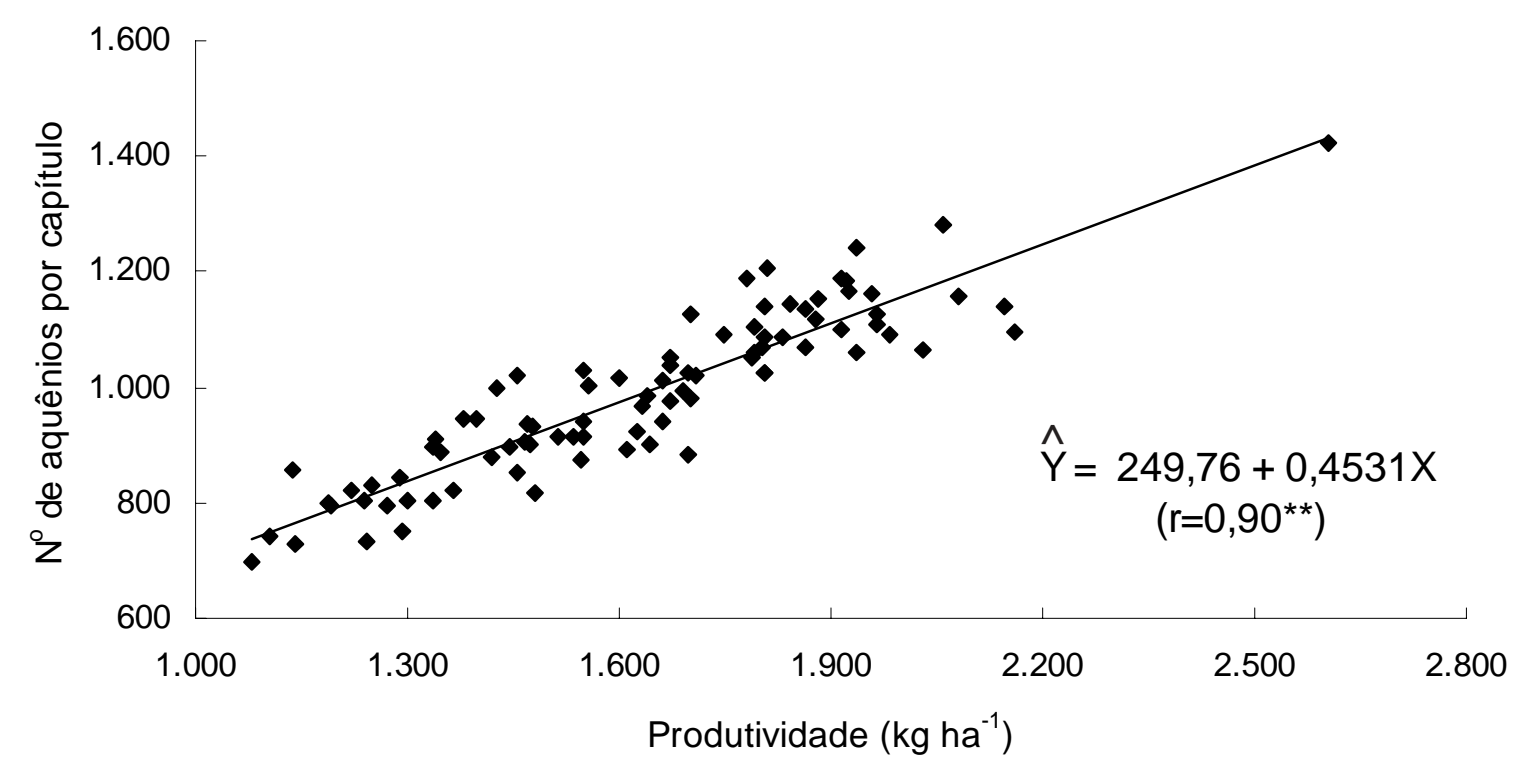

Figura 2 - Correlação entre a produtividade do girassol e o número de aquênios por capítulo. Embrapa Soja, Londrina-PR, 2000.

Tabela 3 - Coeficientes de correlação e equações para produtividade, número de aquênios por capítulo e diâmetro do capítulo. Embrapa Soja, 2000. Londrina-PR

\begin{tabular}{|l|c|c|}
\hline \multicolumn{1}{|c|}{ Correlações } & Coeficiente de correlação $(\mathrm{r})$ & Equação \\
\hline Produtividade $\mathrm{x} \mathrm{n} \mathrm{n}^{\mathrm{o}}$ aquênios/capítulo & 0,90 & $\hat{\mathrm{Y}}=249,76+0,4531 \mathrm{X}$ \\
Produtividade $\mathrm{x}$ diâmetro do capítulo & 0,81 & $\hat{\mathrm{Y}}=8,56+0,0045 \mathrm{X}$ \\
Diâmetro do capítulo x $\mathrm{n}^{\mathrm{o}}$ aquênios/capítulo & 0,68 & $\hat{\mathrm{Y}}=8,32+0,0076 \mathrm{X}$ \\
\hline
\end{tabular}


Tabela 4 - Percentagem de controle da trapoeraba, do picão-preto, do amendoim-bravo, da corda-de-viola e percentagem de controle geral aos 25 e 35 DAA, em função dos tratamentos. Embrapa Soja, Londrina-PR, 2000

\begin{tabular}{|c|c|c|c|c|c|c|c|c|c|c|}
\hline \multirow{2}{*}{ Tratamentos } & \multicolumn{2}{|c|}{ Trapoeraba } & \multicolumn{2}{|c|}{ Picão-preto } & \multicolumn{2}{|c|}{ Amendoim-bravo } & \multicolumn{2}{|c|}{ Corda-de-viola } & \multicolumn{2}{|c|}{ Controle Geral } \\
\hline & 25 & 35 & 25 & 35 & 25 & 35 & 25 & 35 & 25 & 35 \\
\hline ACE & $98 \mathrm{a}$ & $99 \mathrm{a}$ & $99 \mathrm{a}$ & $99 \mathrm{a}$ & $58 \mathrm{c}$ & $57 \mathrm{c}$ & $99 \mathrm{a}$ & $99 \mathrm{a}$ & 98 a & $97 \mathrm{a}$ \\
\hline $\mathrm{ACE}+\mathrm{BO}$ & $97 \mathrm{a}$ & $97 \mathrm{a}$ & $96 \mathrm{a}$ & $96 \mathrm{a}$ & $60 \mathrm{c}$ & $58 \mathrm{c}$ & $97 \mathrm{a}$ & $96 \mathrm{a}$ & $96 \mathrm{a}$ & $96 \mathrm{a}$ \\
\hline $\mathrm{ACE}+\mathrm{AB}$ & 99 a & $98 \mathrm{a}$ & $98 \mathrm{a}$ & $98 \mathrm{a}$ & $58 \mathrm{c}$ & $52 \mathrm{c}$ & $98 \mathrm{a}$ & $98 \mathrm{a}$ & $98 \mathrm{a}$ & $96 \mathrm{a}$ \\
\hline OXY & $62 \mathrm{c}$ & $71 \mathrm{bc}$ & $54 \mathrm{bc}$ & $55 \mathrm{~b}$ & $97 \mathrm{a}$ & $95 \mathrm{a}$ & $68 \mathrm{~b}$ & $68 \mathrm{~b}$ & $61 \mathrm{c}$ & $68 \mathrm{bc}$ \\
\hline $\mathrm{OXY}+\mathrm{BO}$ & $65 \mathrm{bc}$ & $69 \mathrm{bc}$ & $48 \mathrm{c}$ & $51 \mathrm{~b}$ & $94 \mathrm{a}$ & $94 \mathrm{a}$ & $56 \mathrm{c}$ & $56 \mathrm{c}$ & $57 \mathrm{c}$ & $67 \mathrm{c}$ \\
\hline $\mathrm{OXY}+\mathrm{AB}$ & $59 \mathrm{c}$ & $64 \mathrm{c}$ & $51 \mathrm{bc}$ & $52 \mathrm{~b}$ & $97 \mathrm{a}$ & $96 a$ & $57 \mathrm{bc}$ & $57 \mathrm{bc}$ & $58 \mathrm{c}$ & $67 \mathrm{c}$ \\
\hline SUL & $75 \mathrm{~b}$ & $75 \mathrm{bc}$ & $62 \mathrm{~b}$ & $60 \mathrm{~b}$ & $78 \mathrm{~b}$ & $76 \mathrm{~b}$ & $90 \mathrm{a}$ & $92 \mathrm{a}$ & $76 \mathrm{~b}$ & $76 \mathrm{~b}$ \\
\hline $\mathrm{SUL}+\mathrm{BO}$ & $76 \mathrm{~b}$ & $79 \mathrm{~b}$ & $62 \mathrm{~b}$ & $60 \mathrm{~b}$ & $77 \mathrm{~b}$ & $78 \mathrm{~b}$ & $94 \mathrm{a}$ & $95 \mathrm{a}$ & $72 \mathrm{~b}$ & $77 \mathrm{~b}$ \\
\hline $\mathrm{SUL}+\mathrm{AB}$ & $75 \mathrm{~b}$ & $76 \mathrm{bc}$ & $61 \mathrm{bc}$ & $59 \mathrm{~b}$ & $78 \mathrm{~b}$ & $76 \mathrm{~b}$ & $90 \mathrm{a}$ & $90 \mathrm{a}$ & $71 \mathrm{~b}$ & $77 \mathrm{~b}$ \\
\hline TRI & $0 \mathrm{~d}$ & $0 \mathrm{~d}$ & $0 \mathrm{~d}$ & $0 \mathrm{c}$ & $0 \mathrm{~d}$ & $0 \mathrm{~d}$ & $0 \mathrm{~d}$ & $0 \mathrm{~d}$ & $10 \mathrm{~d}$ & $14 \mathrm{~d}$ \\
\hline $\mathrm{TRI}+\mathrm{BO}$ & $0 \mathrm{~d}$ & $0 \mathrm{~d}$ & $0 \mathrm{~d}$ & $0 \mathrm{c}$ & $0 \mathrm{~d}$ & $0 \mathrm{~d}$ & $0 \mathrm{~d}$ & $0 \mathrm{~d}$ & $10 \mathrm{~d}$ & $14 \mathrm{~d}$ \\
\hline $\mathrm{TRI}+\mathrm{AB}$ & $0 \mathrm{~d}$ & $0 \mathrm{~d}$ & $0 \mathrm{~d}$ & $0 \mathrm{c}$ & $0 \mathrm{~d}$ & $0 \mathrm{~d}$ & $0 \mathrm{~d}$ & $0 \mathrm{~d}$ & $10 \mathrm{~d}$ & $14 \mathrm{~d}$ \\
\hline TSC & $0 \mathrm{~d}$ & $0 \mathrm{~d}$ & $0 \mathrm{~d}$ & $0 \mathrm{c}$ & $0 \mathrm{~d}$ & $0 \mathrm{~d}$ & $0 \mathrm{~d}$ & $0 \mathrm{~d}$ & $0 \mathrm{e}$ & $0 \mathrm{e}$ \\
\hline $\mathrm{TSC}+\mathrm{BO}$ & $0 \mathrm{~d}$ & $0 \mathrm{~d}$ & $0 \mathrm{~d}$ & $0 \mathrm{c}$ & $0 \mathrm{~d}$ & $0 \mathrm{~d}$ & Od & $0 \mathrm{~d}$ & $0 \mathrm{e}$ & $0 \mathrm{e}$ \\
\hline $\mathrm{TSC}+\mathrm{AB}$ & $0 \mathrm{~d}$ & $0 \mathrm{~d}$ & $0 \mathrm{~d}$ & $0 \mathrm{c}$ & $0 \mathrm{~d}$ & $0 \mathrm{~d}$ & $0 \mathrm{~d}$ & $0 \mathrm{~d}$ & $0 \mathrm{e}$ & $0 \mathrm{e}$ \\
\hline $\mathrm{TC}$ & $100 \mathrm{a}$ & $100 \mathrm{a}$ & $100 \mathrm{a}$ & $100 \mathrm{a}$ & $100 \mathrm{a}$ & $100 \mathrm{a}$ & $100 \mathrm{a}$ & $100 \mathrm{a}$ & $100 \mathrm{a}$ & $100 \mathrm{a}$ \\
\hline $\mathrm{TC}+\mathrm{BO}$ & $100 \mathrm{a}$ & $100 \mathrm{a}$ & $100 \mathrm{a}$ & $100 \mathrm{a}$ & $100 \mathrm{a}$ & $100 \mathrm{a}$ & $100 \mathrm{a}$ & $100 \mathrm{a}$ & $100 \mathrm{a}$ & $100 a$ \\
\hline $\mathrm{TC}+\mathrm{AB}$ & $100 \mathrm{a}$ & $100 \mathrm{a}$ & $100 \mathrm{a}$ & $100 \mathrm{a}$ & $100 \mathrm{a}$ & $100 \mathrm{a}$ & $100 \mathrm{a}$ & $100 \mathrm{a}$ & $100 \mathrm{a}$ & $100 \mathrm{a}$ \\
\hline $\mathrm{CV}(\%)$ & 15,56 & 16,78 & 18,45 & 18,87 & 16,72 & 16,69 & 14,96 & 15,10 & 12,12 & 11,80 \\
\hline
\end{tabular}

Médias seguidas pelas mesmas letras, nas colunas, não diferem estatisticamente entre si a 5\% de probabilidade pelo teste de Duncan.

No controle do amendoim-bravo (Euphorbia heterophylla), os tratamentos contendo o herbicida oxyfluorfen foram eficientes, não diferindo da testemunha capinada aos 25 e 35 DAA. Esse herbicida, quando aplicado em condições de pré-emergência, age sobre o hipocótilo, o epicótilo e os meristemas foliares, impedindo a emergência das espécies daninhas (Rodrigues \& Almeida, 1998). Tem como características a baixa solubilidade em água e a baixa mobilidade no perfil do solo, permanecendo numa fina camada superficial. Como a semeadura do girassol é realizada abaixo da faixa onde permanece o produto, a seletividade se dá por posicionamento, não afetando a emergência da cultura.

Os tratamentos com os herbicidas acetochlor e sulfentrazone apresentaram controle mediano do amendoim-bravo em ambas as avaliações. O trifluralin, aplicado isoladamente ou em mistura com as fontes de B, não foi eficiente no controle dessa planta daninha, assemelhando-se à testemunha sem capina.

Os tratamentos com os herbicidas acetochlor e sulfentrazone apresentaram controle da corda-de-viola (Ipomoea grandifolia) acima de 90\%, não diferindo estatisticamente da testemunha capinada. Brighenti et al. (2000a), trabalhando com a mesma dosagem de sulfentrazone, verificaram controle da corda-de-viola em torno de 90\%. Esses níveis de controle demonstram não só a eficiência do herbicida no controle dessa espécie, como também a possibilidade da mistura com as duas fontes de B. Para os tratamentos com oxyfluorfen isolado e em combinação com as fontes de $\mathrm{B}$, ocorreu menor percentagem de controle.

O acetochlor é eficiente no controle da trapoeraba, do picão-preto e da corda-de-viola e pouco eficiente no controle do amendoim-bravo. Entretanto, o oxyfluorfen é eficiente no 
controle do amendoim-bravo e pouco eficiente no controle das outras três espécies. Assim, em áreas de infestação mista de trapoeraba, de picão-preto, de amendoim-bravo e de corda-de-viola, a combinação de acetochlor e oxyfluorfen aumentaria o espectro de ação sobre essas invasoras.

Nas avaliações do controle geral das plantas daninhas realizadas aos 25 e 35 DAA, os tratamentos $\mathrm{ACE}, \mathrm{ACE}+\mathrm{BO}$ e $\mathrm{ACE}+\mathrm{AB}$ foram eficientes no controle das invasoras e superiores estatisticamente a todos os demais. Para os tratamentos com os herbicidas oxyfluorfen e sulfentrazone, isolados e em mistura com as fontes de $\mathrm{B}$, houve controle mediano das espécies daninhas próximo a $70 \%$. Os tratamentos contendo o herbicida trifluralin apresentaram aumento do controle geral das plantas daninhas, passando de $10 \%$, na primeira avaliação, para $14 \%$, na segunda avaliação; entretanto, foram inferiores a todos os demais, exceto a testemunha sem capina.

Com base nos resultados apresentados, conclui-se que é viável a aplicação de boro junto à calda de herbicidas em pré-emergência na cultura do girassol. A combinação dos herbicidas com o ácido bórico resulta em solução mais homogênea da calda de pulverização, quando comparada com os herbicidas mais bórax. A produtividade da cultura e os componentes de produção (número de aquênios por capítulo, peso de mil aquênios e diâmetro de capítulo) do tratamento acetochlor + ácido bórico indicam a maior eficiência desse tratamento na aplicação de boro junto à calda de herbicidas para a cultura do girassol. O herbicida acetochlor, aplicado isoladamente ou em mistura com as duas fontes de boro, foi eficiente no controle da trapoeraba, do picão-preto e da corda-de-viola, sendo o tratamento mais eficiente no controle das plantas daninhas. $\mathrm{O}$ herbicida oxyfluorfen, aplicado isoladamente ou em mistura com as duas fontes de boro, foi eficiente no controle do amendoim-bravo. O herbicida sulfentrazone, aplicado isoladamente ou em mistura com as duas fontes de boro, foi eficiente no controle da corda-de-viola.

\section{AGRADECIMENTOS}

Aos técnicos agrícolas da Embrapa Soja Mário Nakano e Esmael da Silva e ao auxiliar de operações Valdenir Saldeira, pelos trabalhos de condução do experimento.

\section{LITERATURA CITADA}

ANDERSON, O. E.; OHKI, K. Cotton growth response and B distribution from foliar application of B. Agron. J., v. 64, n. 5, p. 665-667, 1972.

BLAMEY, F. P. C.; MOULD, D.; CHAPMAN, J. Critical boron concentrations in plant tissue of two sunflower cultivars. Agron. J., v. 71, n. 2, p. 243-247, 1979.

BLAMEY, F. P. C.; ZOLLINGER, R. K.; SCNEITER, A. A. Sunflower production and culture. In: SCNEITER, A.A. (Ed). Sunflower technology and production. Madison: Wisconsin, American Society of Agronomy, 1997. p. 595-670, (Agronomy, 35).

BRIGHENTI, A. Expansão do girassol e os problemas no controle das plantas daninhas. R. Bras. Herbic., v. 1, n. 3, 2000. (Nota técnica).

BRIGHENTI, A. M. et al. Controle químico de plantas daninhas na cultura do girassol em solo de textura argilosa. R. Bras. Herb., v. 1, n. 1, p. 85-88, 2000a.

BRIGHENTI, A. M. et al. Sunflower tolerance to preemergence broadleaf weed herbicides. In:

INTERNATIONAL SUNFLOWER CONFERENCE, 15, 2000, Toulouse. Proceedings... Toulouse: 2000b. T. 2, p. C-73-77.

CALLE-MANZANO, C. L. Carencia de boro en girasol. Madrid: Ministerio de Agricultura, Pesca Y Alimentacion, 1985. 12 p. (Hojas Divulgadoras, 7).

CASTRO, C. et al. A cultura do girassol. Londrina: Empresa Brasileira de Pesquisa Agropecuária - CNPSo. 1997. 36 p. (EMBRAPA - CNPSo. Circular Técnica, 13).

CONNOR, D. J.; HALL, A. J. Sunflower physiology. In: SCHNEITER, A.A. (Ed.) Sunflower technology and production. 2.ed. Madison: American Society of Agronomy, 1997. p. 113-182. (Agronomy, 35).

EMPRESA BRASILEIRA DE PESQUISA AGROPECUÁRIA - EMBRAPA. Informe da avaliação de genótipos de girassol, 1998/99 e 1999. Londrina: 1999. 99 p. (Embrapa Soja. Documentos, 137).

GIL MARTINEZ, F. Elementos de fisiología vegetal: relaciones hídricas, nutrición mineral, transporte, metabolismo. Madrid: Ediciones Mundi-Prensa, 1995. p. $249-283$. 
GUPTA, U. C. Boron and its role in crop production. Boca Raton: CRC Press, 1993. 236 p.

MORTVEDT, J. J.; WOODRUFF J. R. Technology and application of boron fertilizers for crops. In: GUPTA, U. C. (Ed). Boron and its role crop production. Boca Raton: CRC Press, 1993. p. 157-176.

REUTER, D. J.; EDWARDS, D. G.; WILHELM, N. S. Temperate and tropical crops. In: REUTER, D.J.; ROBINSON, J.B. (Eds.). Plant analysis. Melbourne: Inkata Press, 1997. p. 83-278.
RODRIGUES, B. N.; ALMEIDA, F. S. Guia de herbicidas. 4.ed. Londrina: 1998. 648 p.

SFREDO, G. J.; SARRUGE, J. R. Acúmulo de micronutrientes em plantas de girassol. Pesq. Agropec.

Bras., v. 25, n. 4, p. 499-503, 1990.

WEAST, R. C.; ASTLE, M. J. (Eds.). CRC handbook of chemistry and physical: a ready-reference book of chemical and physical data. 62.ed. Boca Raton: CRC, 1982. p. B84, B146. 